\title{
Multi-Platform Controller Interface for SCADA Application *
}

\author{
José Duarte* Luís Rato* Paulo Shirley ${ }^{* *}$ Manuel Rijo ${ }^{* * *}$ \\ * Departamento de Informática - Universidade de Évora, Rua Romão \\ Ramalho 59, 7000-671 Évora, Portugal (m5642@alunos.uevora.pt, \\ lmr@di.uevora.pt) \\ ** DCeT - Universidade Aberta, Rua Fernão Lopes, 9, $1^{\circ}$ Esq. \\ 1000-132 Lisboa,Portugal (pos@univ-ab.pt) \\ *** NuHCC - Núcleo de Hidráulica e Controlo de Canais - \\ Universidade de Évora - Pólo da Mitra Apartado 94, 7002-554 Évora \\ Portugal (rijo@uevora.pt)
}

\begin{abstract}
This paper concerns the development of a SCADA-Controller Interface (SCI) application for an open-channel experimental facility. Water delivery canals are complex and spatially distributed systems. The proposed application is to be applied to test control algorithms developed by several research groups with different technical approaches. The proposed interface allows the development of controllers in different environments - $\mathrm{C} / \mathrm{C}++$, MATLAB/Simulink, and GNU Prolog - and may be easily extended to other environments. The experimental facilities with the used instrumented canal, the programmable logic controller (PLC) network and the SCADA system are also described in this paper. Finally, some software experimental results are presented.
\end{abstract}

Keywords: Multi-platform, Data acquisition, Control algorithms, Efficiency enhancement, Real-time communication, Supervisory control

\section{INTRODUCTION}

Management of water delivery systems is increasingly important as fresh water scarcity is becoming more and more a critical issue in many places worldwide. Thus an efficient management of water delivery canals, that minimizes water losses and/or energy consumption is a main issue.

Nevertheless, this management task brings about conflicting goals - water losses vs. service quality. On one side, users demand more and more flexibility on water delivery. On the other side, the use of the traditional water supplyoriented delivery schedules, like rotation (Almeida et al. (2002)) may attain a very low level of water losses but high price in terms quality of service of water delivery.

Modern water delivery canals with sensors, automatic actuators, communication networks and the application of advanced control techniques may have an important role on the improvement of the water savings and water delivery service.

Several approaches have followed in order to address these questions, such as: distant vs. local control strategies, adaptive control, predictive control, and optimal control (Rijo (1999); Litrico and Fromion (2003); Shirley et al. (2007); Aguilar et al. (2009); Lemos et al. (2009)). A knowledge based system implemented in a declarative

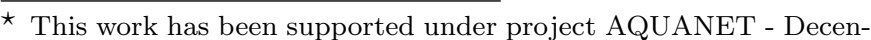
tralized and Reconfigurable Control for Water delivery Multipurpose Canal Systems (PTDC/EEA-CRO/102102/2008).
}

language as Prolog is another possible approach (Rato et al. (2005)).

These control approaches have been tested in the instrumented large scale experimental canal of the NuHCC Hydraulics and Canal Control Centre of the University of Évora, using several developed software packages that communicates to the Supervisory Control and Data Acquisition (SCADA) system. These approaches have been developed on different programming environments such as $\mathrm{C} / \mathrm{C}++$, Visual Basic, and Matlab and resulted in non compatible software packages

The interface presented in this paper aims to establish a common framework for control algorithm development at this experimental plant.

\section{WATER CANAL DESCRIPTION}

This project is being developed at the NuHCC (Fig. 1). NuHCC activity began in 2001 and was financed by the European Union (Program INTERGET IIC).

The canal used has a trapezoidal lined cross section geometry and is constituted by four pools of roughly $40 \mathrm{~m}$ each, resulting in a $145 \mathrm{~m}$ long instrumented canal. The facility also includes a traditional water delivery canal that completes a closed water circuit. The canal inflow is manipulated by an electrical controlled MONOVAR valve. The water depth profiles along the four pools may be controlled by three sluice gates and one overshot gate (vertical). Upstream of each gate there is an offtake that 


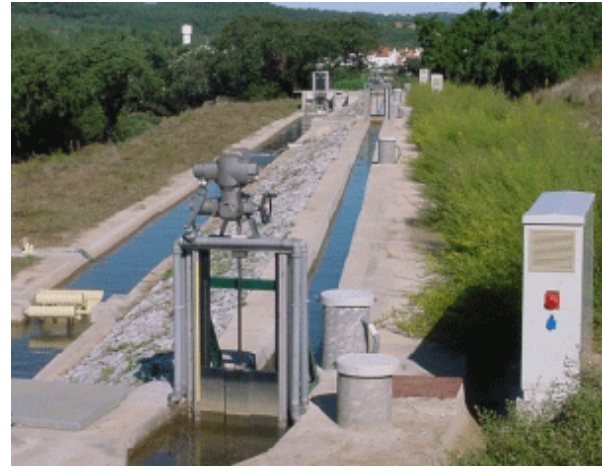

Fig. 1. Experimental water canal at the University of Évora

feeds directly the traditional local upstream controlled canal. Each offtake is equipped with a flowmeter and an electrical butterfly valve. Concerning sensors, there are three float and counter-weight water level sensors in stilling wells for each pool - one at each end and another one in the middle of the pool. Fig. 2 shows a schematic diagram of the experimental plant. This facility is described in more detail in Ratinho et al. (2002) and Almeida et al. (2002).

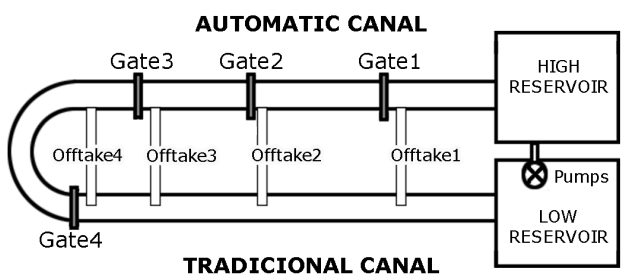

Fig. 2. Schematic diagram of the experimental water canal at the University of Évora

\subsection{Data acquisition and Supervision System}

The experimental facility is also equipped with a network of 6 PLCs. Five local PLCs (one for each gate and one for the intake valve) and one central master PLC. The data acquisition and analog-to-digital conversion are performed locally at each PLC. These are interconnected by a MODBUS network to the master PLC, that communicates to the SCADA computer by a serial port RS232 interface. See Fig. 3.

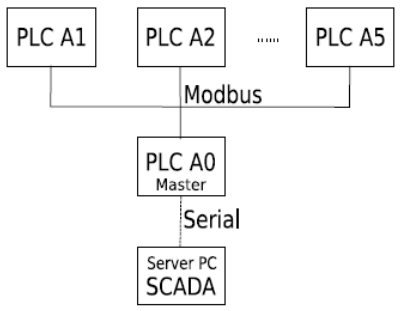

Fig. 3. Schematic diagram of the PLC network and SCADA PC server.

The SCADA system is built over the WIZCON environment, that presents a user friendly graphical interface (Fig. 4) to command the process as well as to observe the evolution of the measured variables over time. The environment runs only on Windows Operating System.

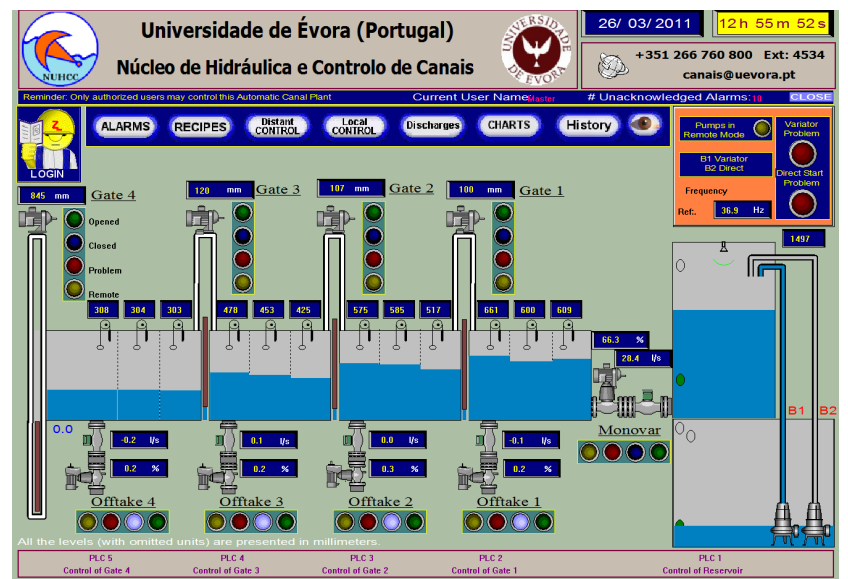

Fig. 4. Main screen of SCADA graphical interface at the SCADA PC server

\section{SCADA-CONTROLLER INTERFACE (SCI) APPLICATION}

The developed SCI application implements a client-server architecture with one server in the SCADA computer and several clients. The clients and server may communicate by TCP/IP (Kozierok (2005)) connection over a computer network on separate hardware, but both may run in the same computer. The computers of this network may run different Operating Systems. In order to cope with this multiplatform environmemnt, several clients were created in $\mathrm{C} / \mathrm{C}++$, Prolog, and Matlab environments.

These (controller-side) clients communicate with the server by TCP/IP connection (Fig. 5), while the server communicates with the SCADA environment by Dynamic Data Exchange (DDE). This scheme is represented in Fig. 6.

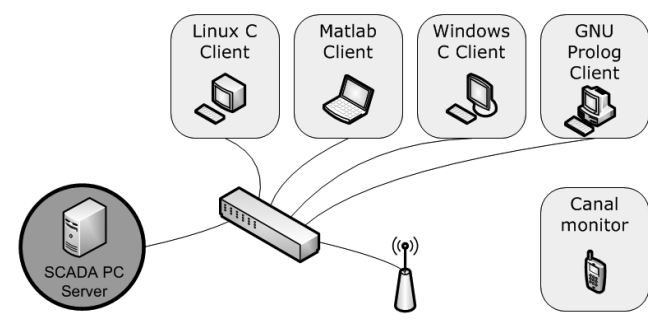

Fig. 5. System network

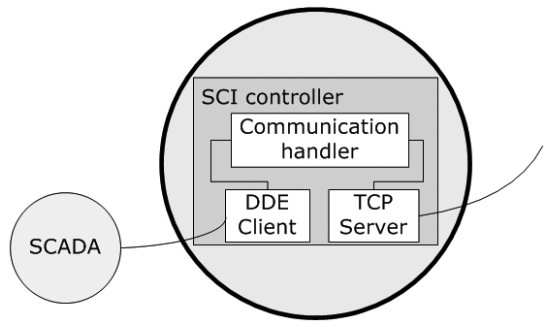

Fig. 6. Controller Server architecture

The major benefit of this system architecture is to allow multi-platform controller side tests. At the present 
moment, the users of the experimental plant can develop their controllers among Windows and Linux, and choose the programming environmemnt: C (Kernighan and Ritchie (1988)), Prolog (Diaz (2009)) or Matlab (Chapman (2008)). Other programming environments such as Java, C\#, and Python will be developed.

\subsection{Server}

The server that the SCI needs to run on the SCADA PC. Since this implies that it must run on a Windows machine, so the server module was developed on ANSI C for Windows.

The server module implements three components: TCP server, communication handler and DDE Client. The module architecture can been seen in Fig. 6 .

TCP Server This module waits for connections from the clients and then sends and receives TCP messages. The server is ready to answer to any requests from any SCI Client, even from different platforms. In order to ensure that, a communication protocol was created. This protocol defines the format and a set of rules for exchanging all messages. This set of rules includes a list of keywords (tags) to easy reference SCADA variables. A partial list of tags is presented in Table 1.

Table 1. Tags examples

\begin{tabular}{c||cl} 
Code & Tag & Meaning \\
\hline \hline 0 & SCD_Q0 & Monovar flow. \\
1 & SCD_D1 & Offtake1 flow. \\
2 & SCD_D2 & Offtake2 flow variable. \\
9 & SCD_M1 & Pool1 upstream water level. \\
14 & SCD_C2 & Pool2 central water level. \\
19 & SCD_J3 & Pool3 downstream water level. \\
50 & SCD_Q0_PER & Monovar opening in percentage. \\
\hline
\end{tabular}

The client can request a set of actions of the same type each time it sends a message to the server (e.g. the request to read four different variables can be done with one command). The message used on the client server TCP/IP connection has the following format:

number_of_actions, action 1 , action2, . . actionN\#

Example: If the client wants to read the monovar, offtake 1, offtake 2 and offtake 4 flow variables, the message should be (before encoding):

[SCD_Q0, SCD_D1, SCD_D2, SCD_D4]

After encoding the message will be:

$4,0,1,2,4 \#$

DDE Client The SCADA system has a DDE communication interface. It was the chosen process of interaction between the SCI Server and the SCADA system.

The SCADA interacts with the master PLC (via a serial link) setting and reading variables are communicated to the slave PLCs via the ModBus network. These variables are identified by tags and represent a state of the controlled plant, includes: sensor measures, setpoints and gains for the local controllers and operation modes.
The Controller Server implements a DDE Client that connects to the SCADA DDE Server and allows to read and write those variables through DDE. To ensure that, three functions were defined:

- DDEInit("WIZCON", "GATE") to establish clientserver DDE connection.

- DDERequest(char var[]) to read values.

- DDEPoke(char [Jvar, char [Jvalor) to write values.

However, while reading usually needs just one call to DDERequest(), to define a command into the actuators several calls of DDEPoke(...) are usually necessary, stating the control mode of one or more (cascade) loops and defining the variable value.

For example, the code to command gate 1 opening setpoint (tag "SCD_U1_SP", code "105") is

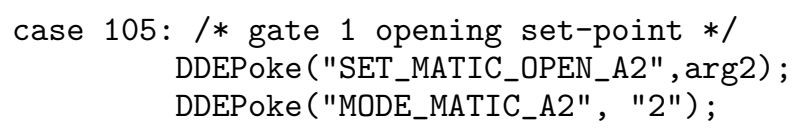

Communication handler This module works like a language interpreter of the SCI Server. It must handle the requests and responses between TCP server and the DDE client.

\subsection{Clients}

The main objective of the client system is to allow the interaction with SCADA using any Operating Systems or programming languages. Several interface clients (ICs) have already been developed and much more can be implemented. At this moment, four ICs are available and fully tested: ANSI C for Windows, ANSI C for Linux, GNU Prolog and Matlab. Each ICs implements a couple of functions to interact with SCADA: scd_read and scd_write.

- scd_read - Send a request to the SCI server to read a list of SCADA variables.

- scd_write - Send a request to the SCI server to write/change a list of variables.

The user can use, in his code or simulation (Matlab/Simulink), any of these functions to read or write values of SCADA variables.

A stub to read four SCADA variables in ANSIC (Windows or Linux) is

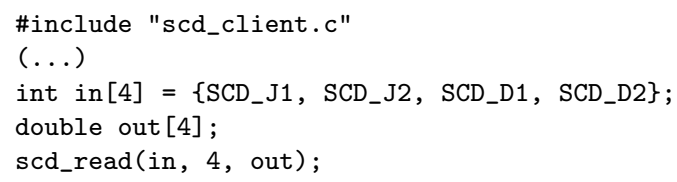

First of all, a list of tags to read should be created and then make the read request. In this case, the variable out will keep the four requested values.

Similarly, in gprolog the equivalent stub would be

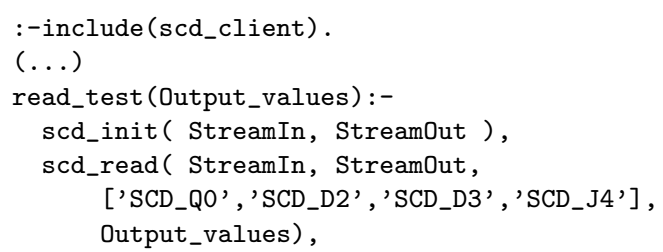


scd_end(StreamIn, Stream0ut).

The predicate read_test(?Output_values) starts by initializing the connection with the SCI server, doing the read request and finally end the connection. The variable Output_values will hold a list with the four values requested.

In Matlab, a single function call can be used to read the same four variables,

ouput = scd_read (['SCD_Q0' ; SCD_D2' ; 'SCD_D3' ; 'SCD_J4'] ) ;

where the variable output holds the four requested values.

\subsection{Canal monitor for cellphones}

A canal monitor (Fig. 7) has been developed for Symbian 60 cellphones using the SCI. Using an wireless connection and a cellphone, it is possible to read several SCADA variables. This feature is very usefull on long controlling tests, because it allows the researcher to check anyware if everything is working as expected.

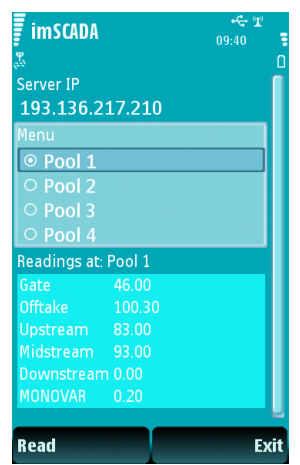

Fig. 7. Canal monitor for cellphones

\section{LATENCY TESTS}

Several tests were made in order to evaluate the latency of the read and write operations i.e. time delay between the operation request and result retrieval. Table 2 , presents the test results. These tests were defined based on the most frequently used commands. For each test, 600 samples were taken with a period of 1 second. All tests were performed on a Intel(R) Core(TM)2 Duo CPU P8700 (2.53Ghz), 4GB of RAM, with an Ethernet connection 10/100 Mbits.

Table 2. List of the lantency tests

\begin{tabular}{c||ccl} 
& Type & \# variables & Description \\
\hline \hline T1 & Read & 1 & Monovar \\
T2 & Read & 2 & Monovar + Reservoir \\
T3 & Read & 4 & Gates \\
T4 & Read & 8 & Gates + Offtakes \\
T5 & Read & 12 & Water levels \\
T6 & Read & 16 & Water levels + Offtakes \\
T7 & Read & 20 & Water levels + Offtakes + Gates \\
\hline T8 & Write & 1 & Monovar \\
T9 & Write & 4 & Gates \\
T10 & Write & 8 & Gates + Offtakes \\
\hline
\end{tabular}

It was decided to run these tests in MATLAB and $\mathrm{C}$ because these are the main tools used by the researchers of the ongoing project. Tables 3 and 4 shows all reading test in $\mathrm{C}$ and Matlab respectively.
Table 3. Readings tests using $\mathrm{C}$ for Windows.

(Values in milliseconds)

\begin{tabular}{l||ccccccc} 
& $\mathrm{T} 1$ & $\mathrm{~T} 2$ & $\mathrm{~T} 3$ & $\mathrm{~T} 4$ & $\mathrm{~T} 5$ & $\mathrm{~T} 6$ & $\mathrm{~T} 7$ \\
\hline \hline Mean & 3.1 & 3.2 & 3.4 & 4.0 & 4.1 & 4.4 & 4.8 \\
Median & 3.0 & 3.0 & 3.0 & 4.0 & 4.0 & 4.0 & 5.0 \\
Mode & 3 & 3 & 3 & 4 & 4 & 4 & 5 \\
Std. Deviation & 0.5 & 0.6 & 0.6 & 0.9 & 0.7 & 1.3 & 1.0 \\
\hline Maximun & 12 & 12 & 11 & 22 & 18 & 31 & 24 \\
Minimun & 2 & 2 & 2 & 2 & 2 & 3 & 3 \\
Range & 10 & 10 & 9 & 20 & 16 & 28 & 21 \\
\hline Percentile 25 & 3 & 3 & 3 & 4 & 4 & 4 & 5 \\
Percentile 50 & 3 & 3 & 3 & 4 & 4 & 4 & 5 \\
Percentile 75 & 3 & 3 & 4 & 4 & 4 & 5 & 5 \\
\hline
\end{tabular}

Table 4. Readings tests using MATLAB. (Values in milliseconds)

\begin{tabular}{l||ccccccc} 
& $\mathrm{T} 1$ & $\mathrm{~T} 2$ & $\mathrm{~T} 3$ & $\mathrm{~T} 4$ & $\mathrm{~T} 5$ & $\mathrm{~T} 6$ & $\mathrm{~T} 7$ \\
\hline \hline Mean & 14.9 & 23.0 & 34.7 & 45.5 & 55.3 & 65.8 & 76.1 \\
Median & 15.0 & 23.0 & 37.0 & 45.0 & 55.0 & 65.0 & 73.5 \\
Mode & 15 & 23 & 38 & 42 & 55 & 63 & 70 \\
Std. Devia- & 2.3 & 2.1 & 4.9 & 7.2 & 6.7 & 8.4 & 9.5 \\
tion & & & & & & & \\
\hline Maximun & 47 & 37 & 46 & 71 & 85 & 109 & 124 \\
Minimun & 6 & 9 & 14 & 21 & 31 & 39 & 56 \\
Range & 41 & 28 & 32 & 50 & 54 & 70 & 68 \\
\hline Percentile 25 & 15 & 23 & 31 & 41 & 51 & 61 & 70 \\
Percentile 50 & 15 & 23 & 37 & 45 & 55 & 65 & 74 \\
Percentile 75 & 15 & 23 & 38 & 49 & 59 & 69 & 81 \\
\hline
\end{tabular}

In Tables 5 and 6 , are shown all writing tests in $\mathrm{C}$ and Matlab respectively.

Table 5. Writing tests using $\mathrm{C}$ for Windows. (Values in milliseconds)

\begin{tabular}{l||ccc} 
& T8 & T9 & T10 \\
\hline \hline Mean & 50.8 & 215.9 & 432.3 \\
Median & 50.0 & 211.0 & 429.0 \\
Mode & 39 & 207 & 445 \\
Std. Deviation & 15.9 & 22.0 & 19.1 \\
\hline Maximun & 87 & 278 & 473 \\
Minimun & 30 & 176 & 402 \\
Range & 57 & 102 & 71 \\
\hline Percentile 25 & 39 & 199 & 418 \\
Percentile 50 & 50 & 211 & 429 \\
Percentile 75 & 63 & 231 & 445 \\
\hline
\end{tabular}

Table 6. Writing tests using MATLAB. (Values in milliseconds)

\begin{tabular}{l||ccc} 
& T8 & T9 & T10 \\
\hline \hline Mean & 58.1 & 254.5 & 484.6 \\
Median & 57.0 & 254.0 & 483.0 \\
Mode & 58 & 235 & 479 \\
Std. Deviation & 11.2 & 20.2 & 29.6 \\
\hline Maximun & 100 & 310 & 560 \\
Minimun & 37 & 200 & 400 \\
Range & 63 & 110 & 160 \\
\hline Percentile 25 & 49 & 239 & 462 \\
Percentile 50 & 57 & 254 & 483 \\
Percentile 75 & 65 & 266 & 508 \\
\hline
\end{tabular}

In the results shown results, the maximum latency is $560 \mathrm{~ms}$, when writing 8 variables (T10 - Matlab), which is compatible with sampling periods of $5 \mathrm{~s}$. This is the usual sampling time value for prototypes and physical models similar to the used canal. (Rato et al. (2007); Aguilar et al. (2009)). 


\section{DATA AQUISITION AND CONTROLLERS TESTS}

This section presents the ongoing research work of two teams using SCI presented in the paper.

\subsection{Example 1 - Calibrating A Finite Dimension Model}

A white box nonlinear simulator for water delivery canals has been tested and validated for the experimental facility here presented using the IC for MATLAB. The SaintVenant equations, used for describing the transport phenomenon, are linearized and then discretized leading to a time discrete state space representation for the canal pool dynamics (Nabais and Botto $(2010,2011)$ ). This pool model has several interesting features namely:

- as the pool model state space is constituted by water depths and discharge along the pool axis, it allows for water depth monitoring along the canal axis (Fig.8(c) and Fig.8(d));

- the pool model accept as boundary conditions discharge and water depth, so the pool model can be used for water conveyance networks as a connection between reservoirs;

- the pool model accounts for discharge perturbations along the pool axis, this way is possible to use the model to develop algorithms for leak detection and localization in open water delivery canals;

- it's simplicity, the Saint-Venant equations are solved through matrices multiplications, with a low computational cost is of particular importance when dealing with large scale systems like the used canal.

In Fig. 8, are presented several water depths for different locations in the canal. The canal was divided in four pools and subjected to variations of the inflow, gate opennings and outflows. Under this severe scenarios, with a large deviation from the stationary configuration, the nonlinear canal simulator captures the systems dynamics and in particular the meaningful water depths have a Variance Accounted For over $88 \%$ to $97 \%$ and a Medium Absolute Error of $15 \mathrm{~mm}$.

The proposed representation is well suitable for model based control strategies namely Model Predictive Control.

\subsection{Example 2 - LQG/LTR and D-SIORHC control}

In order to design controlers, obtained around the desired equilibrium point, with a sampling interval of $1 \mathrm{~s}$. This data is then decimated to the desired controller sampling period, either $2 s$ or $5 s$. After de-trending and filtering data, this is used to identify MIMO linear input/output models relating gate positions and pool water levels (at the desired sections). From these i/o models state-space realizations are then build. For decentralized control, the models have a structure that fits the design algorithm used.

Fig. 9 shows results obtained with the well known discrete time LQG/LTR algorithm for a single pool. Although multivariable LQG/LTR has already been tried with success, these results are shown for the sake of illustration.

In this algorithm, the controller acts to optimize the quadratic cost function
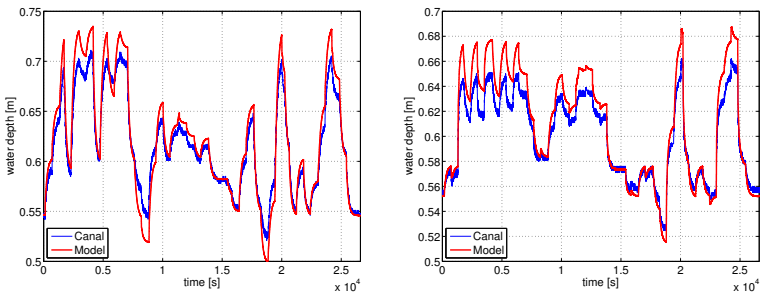

(a) Downstream water level (b) Downstream water level for pool $1, Y_{1}\left(L_{1}, t\right)$.

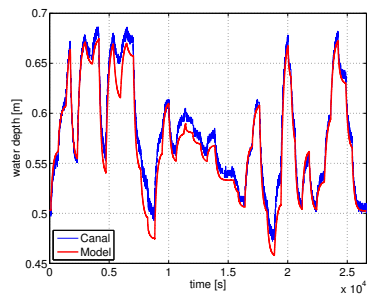
for pool $2, Y_{2}\left(L_{2}, t\right)$

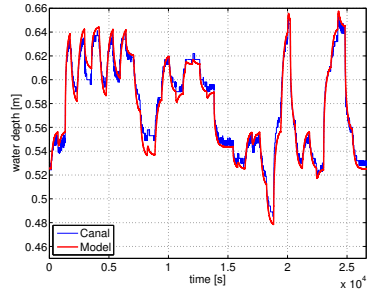

(c) Water depth in the middle (d) Water depth in the middle of pool $1, Y_{1}\left(L_{1} / 2, t\right)$.

of pool $2, Y_{2}\left(L_{2} / 2, t\right)$.

Fig. 8. White box model validation for a open water canal, subjected to upstream discharge, gate movement and water extraction variation.
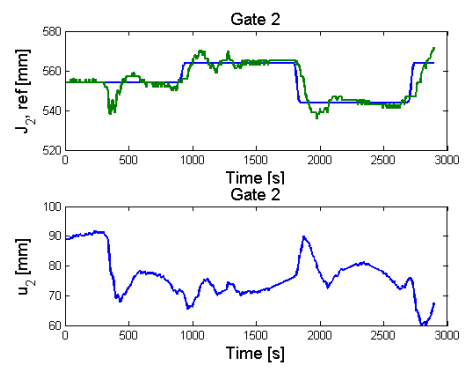

Fig. 9. LQG/LTR algorithm

$$
J=\sum_{t=0}^{\infty}\left(|e(t)|^{2}+\rho|u(t)|^{2}\right)
$$

where $e$ is the tracking error, $u$ is the manipulated variable and $\rho$ is a positive weight. At the instant $300 \mathrm{~s}$, the lateral offtake of the water delivery canal has been opened, causing the gate to close to keep the pool water level close to the setpoint. After that, the setpoint has been increased.

The response shows the typical oscillatory behavior of LQG controllers. This can be avoided either by the increase on the weight $\rho$ (at the cost of making the response sluggish!) or by changing the basic algorithm.

Fig. 10 shows results obtained with D-SIORHC, a distributed version of the SIORHC predictive controller, applied to three canal pools. This is a model predictive control algorithm that minimizes a receding horizon cost under the constraint that the final state vanishes at the end of the prediction horizon. This so-called stability constraint ensures, under certain conditions, that the algorithm yields a stable closed loop for a finite prediction horizon. The distributed version assumes that local pool models interact with adjacent pools via the manipulated variables (gate positions). According to a procedure that resembles a Nash game local controllers negotiate the optimal gate positions such as to reach a consensus. As apparent from Fig. 10, 

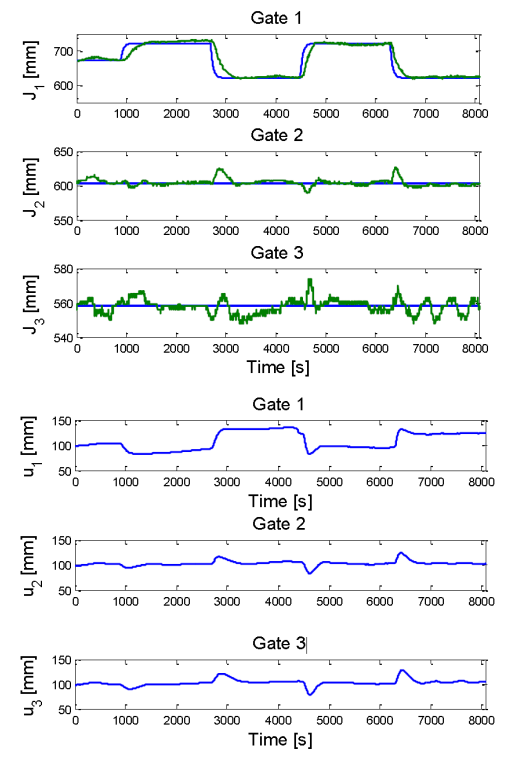

Fig. 10. D-SIORHC predictive controller

the stability constraint leads to a control law that avoids the oscillatory behavior of GPC.

It is remarked that these examples are presented just for the sake of illustration of the use of the IC for MATLAB and that no effort has been presented to optimize results. The point to stress is that this interface allows easy prototyping of controllers for the water delivery canal.

\section{CONCLUSION AND PERSPECTIVES}

A SCADA-Controller Interface was developed and a framework was proposed in order to establish a common interface an automatic experimental water delivery canal. This approach will permit the development of very different control algorithms, implemented in several programing environments and running in two operating systems Gnu/Linux and Windows. This framework is a key step to promote the reuse and comparison of advanced control algorithms in the NuHCC experimental canal.

In a near future, a SCI emulator will be developed. This emulator will implement a TCP Server and a communication handler as well, but it will not implement the DDE Client. This emulator will respond to the ICs, that should not notice the difference between the real system and the emulator from the software point of view. The emulator should also simulate the dynamical behavior of the canal. The main goal of this emulator is to simulate the SCADA application behavior, so that it will provide a first level of debugging for any new controller applications. Thus, when a new control algorithm is proposed, it will be tested in the experimental plant, after successfully passing the emulator test.

\section{ACKNOWLEDGEMENTS}

The results shown in Fig. 9 and 10 concern to research work which has been performed by J. M. Lemos, L. F. Pinto, F. M. Cadete and J. M. Igreja from INESC-ID. The results shown in Fig. 8 concern to research work which has been performed by J. Nabais and M.A. Botto through IDMEC by the Associated Laboratory in Energy, Transports, Aeronautics and Space.

\section{REFERENCES}

Aguilar, J., Langarita, P., Linares, L., and Rodellar, J. (2009). Automatic control of flows and levels in an irrigation canal. Industry Applications, IEEE Transactions on, 45(6), 2198-2208. doi:10.1109/TIA.2009.2031941.

Almeida, M., Figueiredo, J., and Rijo, M. (2002). Scada configuration and control modes implementation on an experimental water supply canal. In 10th Mediterranean Conference on Control and Automation, Lisbon, Portugal.

Chapman, S.J. (2008). MATLAB Programming for Engineers. Cengage Learning, 4 edition.

Diaz, D. (2009). GNU PROLOG - A Native Prolog Compiler with Constraint Solving over Finite Domains. 1.9 edition.

Kernighan, B.W. and Ritchie, D.M. (1988). C Programming Language. Prentice Hall, 2 edition.

Kozierok, C.M. (2005). The TCP/IP Guide: A Comprehensive, Illustrated Internet Protocols Reference. No Starch Press, 1 edition.

Lemos, J.M., Machado, F., Nogueira, N., Rato, L., and Rijo, M. (2009). Adaptive and non-adaptive model predictive control of an irrigation channel, networks and heterogeneous media,. AIMS-American Institute of Mathematical Sciences, Special Issue on Irrigation Channels and Related Problems, Volume: 4, Number: 2, $303-324$.

Litrico, X. and Fromion, V. (2003). Advanced control politcs and optimal performance for an irrigation canal. In Proceedings of the 2003 European control conference, Cambridge, UK.

Nabais, J. and Botto, M.A. (2010). Qualitative comparison between two open water canal models. In 9th Portuguese Conference on Automatic Control, Coimbra, Portugal.

Nabais, J. and Botto, M.A. (2011). Linear model for open water canal pools. In 8th Internation Conference on Informatics in Control, Automation and Robotics, Noordwijkerhout, Netherlands (Submitted).

Ratinho, T., Figueiredo, J., and Rijo, M. (2002). Modelling, control and field tests on an experimental irrigation canal. In MED'2002, 10th Mediterranean Conference on Control and Automation, Lisbon, Portugal.

Rato, L., Rodrigues, I.P., and Gomes, R. (2005). Knowledge representation approach to closed loop control system - a tank system case-study. In J. Filipe, J. AndradeCetto, and J.L. Ferrier (eds.), ICINCO, 113-118. INSTICC Press.

Rato, L., Salgueiro, P., Lemos, J.M., and Rijo, M. (2007). Adaptive predictive controller applied to an open water canal. In J. Zaytoon, J.L. Ferrier, J. Andrade-Cetto, and J. Filipe (eds.), ICINCO-SPSMC, 357-360. INSTICC Press.

Rijo, M. (1999). Local automatic control modes in an experimental irrigation canal. In Irrigation and Drainage System, volume 17, 179-193. Kluwer Academic Pub.

Shirley, P., Lemos, J., Nogueira, N., and Machado, F. (2007). Model analysis and control of a four segment irrigation canal. 1-6. doi:10.1109/MED.2007.4433678. 